\title{
Diffuse low-grade glioma - Changing concepts in diagnosis and management: A review
}

\author{
Rashid Jooma \\ Aga Khan University, rashid.jooma@aku.edu \\ Muhammad Waqas \\ Aga Khan University, muhammad.waqas@aku.edu \\ Inamullah Khan \\ Aga Khan University
}

Follow this and additional works at: https://ecommons.aku.edu/pakistan_fhs_mc_surg_neurosurg

Part of the Neurology Commons, Neurosurgery Commons, and the Surgery Commons

\section{Recommended Citation}

Jooma, R., Waqas, M., Khan, I. (2019). Diffuse low-grade glioma - Changing concepts in diagnosis and management: A review. Asian Journal of Neurosurgery, 14(2), 356-363.

Available at: https://ecommons.aku.edu/pakistan_fhs_mc_surg_neurosurg/190 


\section{Asion Journal of Asian Journal of Neurosurgery \\ Home \\ Current issue \\ Instructions \\ Submit article}

Asian J Neurosurg. 2019 Apr-Jun; 14(2): 356-363.

PMCID: PMC6516028

doi: 10.4103/ajns.AJNS_24_18: 10.4103/ajns.AJNS_24_18

PMID: $\underline{31143247}$

\section{Diffuse Low-Grade Glioma - Changing Concepts in Diagnosis and Management: A Review}

$\underline{\text { Rashid Jooma, Muhammad Waqas, and Inamullah Khan }}$

Department of Surgery, The Aga Khan University Hospital, Karachi, Pakistan

Address for correspondence: Dr. Rashid Jooma, Department of Surgery, The Aga Khan University Hospital, Karachi, Pakistan. E-mail: rashidjooma@gmail.com

Copyright : : 2019 Asian Journal of Neurosurgery

This is an open access journal, and articles are distributed under the terms of the Creative Commons AttributionNonCommercial-ShareAlike 4.0 License, which allows others to remix, tweak, and build upon the work noncommercially, as long as appropriate credit is given and the new creations are licensed under the identical terms.

\section{Abstract}

Though diffuse low-grade gliomas (dLGGs) represent only $15 \%$ of gliomas, they have been receiving increasing attention in the past decade. Significant advances in knowledge of the natural history and clinical diversity have been documented, and an improved pathological classification of gliomas that integrates histological features with molecular markers has been issued by the WHO. Advances in the radiological assessment of dLGG, particularly new magnetic resonance imaging scanning sequences, allow improved diagnostic and prognostic information. The management paradigms are evolving from "wait and watch" of the past to more active interventional therapy to obviate the risk of malignant transformation. New surgical technologies allow more aggressive surgical resections with a reduction of morbidity. Many reports suggest the association of gross total resection with longer overall survival and progression-free survival in addition to better seizure control. The literature also shows the use of chemotherapeutics and radiation therapy as important adjuncts to surgery. The goals of management have has been increasing survival with increasing stress on quality of life. Our review highlights the recent advances in the molecular diagnosis and management of dLGG with trends toward multidisciplinary and multimodality management of dLGG with an aim to surgically resect the primary disease, followed by chemoradiation in cases of progressive or recurrent disease.

Keywords: Astrocytoma, low-grade glioma, oligodendroglioma, seizure, surgical resection

\section{Introduction}

Diffuse low-grade gliomas (dLGGs) are infiltrative but slowly growing intrinsic brain tumors derived from glial cell lines. These neoplasms usually manifest as seizures in young or middle-aged adults who are otherwise well and tend to appear as nonenhancing lesions on computed tomography (CT) or magnetic resonance imaging (MRI) scans, located within or adjacent to eloquent regions of the brain. This clinical scenario leads to medical advice conflicting between observation and intervention being offered to the 
patient. However, information on the natural history and biology of dLGG has accumulated rapidly in the past decade, and the uncertainties of management are being clarified by newer concepts evidenced by epidemiologic, clinical, and genomic studies.

In this review, we intend to critically analyze the present literature regarding the diagnosis, natural history, and management of dLGGs. The review will mainly focus on Grade II astrocytoma and oligodendroglioma, explore the features defining dLGG and the recent advance in brain tumor classification that in addition to the traditional microscopic typing and grading, and incorporate data from molecular biomarkers to provide an integrated histologic-genomic diagnosis. Surgical management and adjunctive chemo-radiation are also being guided and refined by the patient's individual clinical and molecular parameters and these changing concepts are highlighted along with the newer methodologies that enhance the safety of surgical resection.

\section{Methods}

We conducted a literature search using PubMed, Embase, and CinhL databases for low-grade gliomas (LGG). Two of the authors conducted the literature search independently during the month of May, 2017. The focus of search was to find literature most relevant to the diagnoses, natural history, and management of LGGs. In addition, studies on comparison of the various management options were also reviewed. The two authors extracted data separately from the relevant articles. The search results were reviewed by the senior author who wrote the final version of this review.

\section{Results}

\section{Clinical presentation in diffuse low-grade glioma}

Epileptic seizures are the most common presentation, i.e., $72 \%-89 \%$ and range from simple-to-complex seizures with or without secondary generalization.[1, 2$]$ Majority of the presenting seizures are resistant to medical management[]ㅡ and affect the patients' quality of life and cognitive capacity and may cause other complications. $[\underline{4}, \underline{5}]$ The seizures at presentation in dLGG patients define the postoperative potential of seizure continuation and are relevant to prognostication.[6] In neurologically intact patients, presentation with seizures is associated with better prognosis. $[\underline{7}, \underline{8}, \underline{9}, \underline{10}]$ In the largest retrospective series of patients published by Pallud et al. 2013, seizures at diagnosis along with complete resection of tumor were found to be independent predictors of, malignant progression-free survival (PFS) and overall survival (OS) of patients.[10]

Multiple factors predispose dLGG patients to the risk of epileptic activity. In such patients, seizures at presentation are commonly seen in patients younger than 60 years.[11] The tumors' tendency for highly epileptogenic areas of mesiotemporal and insular cortex in addition to frontal, temporal, and parietal lobes explains most of the presentation. Higher frequencies of seizures have been reported with tumors in proximity to the central sulcus.[12] Similarly, oligodendroglioma and oligoastrocytoma which involve the cortex in majority of the cases have a higher susceptibility to epileptic activity compared to astrocytoma, found predominantly in the white matter.[13] Preventing seizures is an important aspect of the multidisciplinary approach in managing dLGG.

dLGGs represent $15 \%$ of gliomas and have the highest incidence in the age group of 35-44 years with increased prevalence in Caucasian males.[14] In cases where seizures are not the initial presentation, progressive tumor growth and infiltration of the adjacent parenchyma leads to neurocognitive changes, bringing the patient to attention due to alteration in mental status $(3-30 \%)[\underline{15}, \underline{16}, \underline{17}]$ and rarely focal neurological deficits $(2 \%-30 \%)[\underline{15}, \underline{16}, \underline{18}]$ or even raised intracranial pressure.[15, 16$]$ In up to $10 \%$ of the cases, the diagnosis is incidental, and the tumor is discovered as a consequence of imaging for head trauma or other unrelated neurological conditions.[19] 


\section{Imaging of diffuse low-grade glioma}

Magnetic Resonance (MR) is the standard of care in dLGG imaging. Though they are by nature diffuse and ill defined, the lesions may be deceptively well circumscribed. dLGG are hyper-intense on T2W images and hypo-intense on T1W images.[20] Fluid-attenuated inversion recovery (FLAIR) tends to show a larger area of signal abnormality than standard T2W images. The extent of the lesion on FLAIR images tends to better correlate with the adequacy of resection margins and is also the most useful in the follow-up of surgical patients to detect recurrence postresection.[21] Contrast enhancement is generally absent but faint and patchy enhancement can be identified up to 60\% of the dLGGs.[22] Among the contrastenhancing dLGGs, majority are identified as oligodendroglioma.[23]

The newer MR sequences and techniques have been widely applied to dLGGs in attempts to predict tumor grade and biological behavior.[24] Magnetic resonance spectroscopy shows a relative preservation of Nacetyl aspartate with an elevated choline peak and without lipid/lactate peaks.

There is however significant overlap between dLGGs and other neoplastic and nonneoplastic lesions.[225]

Tumor histological sampling for examination and genotyping remains the gold standard for diagnosing dLGGs.[26] Apparent diffusion coefficient (ADC) measurements have a high accuracy in differentiating high-grade from LGGs, but however suffer from lack of standardized criteria and methodology.[27] Diffusion tensor imaging (DTI) has been used both in surgical planning[료 ] and in trying to determine tumor grade using diffusivity parameters. $[\underline{29}, \underline{30}] \mathrm{MR}$ perfusion studies reveal near-normal relative cerebral blood volume (rCBV) in LGGs. Changes in rCBV have been used to monitor changes in biological behavior of these lesions.[31] Susceptibility-weighted imaging is exquisitely sensitive to the presence of intra-tumoral hemorrhages, the presence of which tends to mitigate against the lesion being a dLGG. Progression of the dLGG to a higher grade lesion is associated with changes in the MR spectrum, perfusion parameters, as well as ADC values, and these studies are useful in follow-up over time.[20]

On CT, the lesion is usually iso-dense to white matter on noncontrast images and does not demonstrate any significant postcontrast enhancement. Calcifications can be identified in the lesions in up to $20 \%$ of the cases, and the calcifications are particularly indicative of oligodendrogliomas.[르] Hemorrhage is rarely if ever present.

\section{Natural history of diffuse low-grade glioma}

Though dLGG is generally considered a slow-growing benign natured tumor, clinical studies suggest that these are progressive neoplastic lesions, and over $70 \%$ will transform to an anaplastic glioma variant or a secondary glioblastoma within a decade. However, there is considerable variation and unpredictability in the growth potential, and the studies of the Montpellier group allow consideration of the patients in three stages of disease: (i) a presymptomatic stage of unknown duration evidenced by the tumors discovered incidentally,[르 (ii) a symptomatic period of about 7 years after the initial presentation (usually a seizure) during which the patient maybe fully functional if the seizures are controlled,[푸] $]$ and (iii) a transformational stage of 2-3 years of more rapid and clinically overt tumor progression associated with induction of anaplasia.[34] Large series of dLGG assessed on serial MRI scans prior to treatment show that they grow continuously at a rate of about $4 \mathrm{~mm} / \mathrm{year}$.[32] Steady growth at varying rates can be seen in incidentally discovered tumors and in symptomatic ones by serial imaging using appropriate analytic software to allow objective measurement of velocity of tumor diameter expansion. There is no such thing as a static or stable dLGG, and there is an inverse relation between growth rate and survival.

Besides increase in tumor diameter, adverse prognostic features at presentation are patients' age over 40 years, nonseizure symptoms, neurological deficits, larger tumor size particularly in excess of $6 \mathrm{~cm}$ diameter, tumor crossing the midline, and dominance of astrocytic cellular features in tumor histology.[34] 
Of the three histological varieties of dLGG, oligodendrogliomas have a better prognostic outlook than astrocytomas with oligoastrocytomas in between though the latter diagnosis is being altered by application of molecular testing for gene mutation status.

\section{Molecular features in low-grade gliomas}

There have been significant advances in the knowledge about the molecular biology of gliomas along with the development of a series of biomarkers allowing improved diagnosis, prognosis, and prediction of response to treatment. The most valuable of these in practice are the mutation status of the genes regulating the Krebs cycle enzymes, isocitrate dehydrogenase 1 and 2 (IDH1/2), whole-arm co-deletion of chromosome arms 1p and 19q, alterations in tumor protein 53 (TP53), alpha thalassemia/mental retardation syndrome X-linked (ATRX), and telomerase reverse transcriptase (TERT). Mutations of IDH1 are encountered more often than IDH2 and occur in $65 \%-80 \%$ of gliomas. Co-deletion of $1 \mathrm{p}$ and $19 \mathrm{q}$ is characteristic of oligodendroglial tumors. Point mutations in IDH1/2 and co-deletion of $1 \mathrm{p} 19 \mathrm{q}$ delineate subsets of dLGG with distinct biology and clinical behavior.

IDH1 mutation at codon 132 is noted in $>70 \%$ of WHO Grade II and III gliomas,[푸] whereas IDH2 mutation is noticed in up to $6 \%$ of them.[흐 ] Deletion of 1 p36 is reported in up to $18 \%$ of astrocytomas, and $73 \%$ of oligodendrogliomas, while the deletion of $19 q 13.3$ is reported in $38 \%$ of astrocytomas and $73 \%$ of oligodendrogliomas.[흐] Their co-deletion is noted in up to $11 \%$ of astrocytomas and $64 \%$ of oligodendrogliomas. TP53 has been reported as genetic hallmark of low-grade astrocytomas, present in more than $60 \%$ of the cases[37] and occurring only rarely in oligodendrogliomas. 1p19q and IDH 1 and 2 mutations are associated with prolonged survival and better response to chemotherapy (see below). ATRX inactivation is also associated with astrocytic tumors, particularly IDH mutated gliomas $(86 \%)$ and may represent a subset of astrocytomas with improved treatment outcomes.[38] Mutations of the TERT promoter is one of the most common molecular markers in gliomas and is to be found in more than $90 \%$ of IDH mutant and 1p/19q co-deleted oligodendrogliomas.[39]

\section{Classification: Integrating histologic and genetic parameters}

In the previous edition (2007) of the WHO Classification of Tumors of the Central Nervous System, microscopic features were used for cytogenetic typing and malignancy grading. The new edition of 2016 is a major conceptual change from the past, in including molecular biomarkers to the traditional histopathologic features in the categorization of individual dLGGs. These are now grouped together irrespective of origin from astrocytes or oligodendrocytes as they share identifiable genetic mutations and prognostic features. The diagnosis of gliomas by integration of histologic and genotypic features in the new classification greatly improves diagnostic accuracy by reducing the interobserver variation of traditional histology but is dependent on the availability of genomic assays. Tumors not assignable to one of the designated tumor categories or not being subjected to molecular testing are labeled not otherwise specified [iguㅏe 1].

The Cancer Genome Atlas has performed an analysis of 293 untreated dLGG and has been able to define three molecular diagnostic classes more accurately than histological class.[40] The first type was characterized by IDH mutations and 1p/19q co-deletion and showed a strong association with oligodendroglioma histology. Other findings in this type were activating mutations of TERT promoter in $96 \%$ of samples. The patients had favorable clinical outcomes with a median survival of 8 years. The second type had IDH mutations but without $1 \mathrm{p} / 19 \mathrm{q}$ co-deletion or TERT promoter mutations. Rather, inactivation of ATRX and mutation of TP53 (86\% and 94\%, respectively) was found in most of this type, and these findings represent a strong association with astrocytoma. The patients had a median survival of 6.3 years. The third type did not have an IDH mutation (wild-type IDH) and had a spectrum of genetic alterations and disease outcomes more typical of primary glioblastoma. 


\section{Diagnosis and management of diffuse low-grade glioma}

The declaration of a dLGG by a seizure followed by the disclosure of the suspicious lesion by MRI scanning is often a matter of grave concern to the patient, his/her family, and his/her medical advisor. In the absence of any other physical manifestation of intracranial disease and particularly when the lesion is in an eloquent or deep-seated region of the hemisphere, the tendency is to prescribe anticonvulsants and plan to follow-up with serial scans. Definitive therapy is deferred until tumor progression is demonstrated on MRI scan or if clinical features change such as seizure intractability or emergence of headache or neurological deficit. However, the accumulating evidence and experience argue for a more active interventional management at onset and the following are offered in support of such a course:

1. The growth rate of dLGG is variable between patients but does continue at a steady rate of approximately $4 \mathrm{~mm} /$ year,[32] eventually accelerating in the 6 months leading up to the inevitable anaplastic transformation

2. Visual inspection of serial MRI scans of a slow growing glioma for change in tumor size may misleadingly underestimate volume expansion and lead to unnecessary delay and loss of survival benefit from tumor resection and/or chemoradiation due to malignant transformation

3. MRI is not diagnostic for a dLGG. Up to $30 \%$ of nonenhancing intrinsic brain tumors seen on postcontrast MRI may eventually be found to be glioblastoma[41]

4. Patients with dLGG undergoing "upfront" early maximal safe resection have a survival advantage over those undergoing a biopsy followed by watchful waiting.[42] This advantage is sustained even after adjustment for the status of IDH mutation and 1p/19q co-deletion (Jakola 2017)

5. Even when located in putatively eloquent regions of the cerebrum, dLGG rarely present with neurological deficits. The slow tumor expansion allows a plastic reorganization of neural function which is displaced away from the tumor to adjacent brain.[43] The implication of this is that the tumor itself is nonfunctional tissue and even seemingly inoperable lesions can be resected, if the access to and the functional boundaries of, the tumor are defined by image guidance and electrostimulation brain mapping

6. Tissue for diagnosis may come from a gross total resection (GTR) or debulking.[444,45] Larger tissue sample is preferred in order to study the heterogeneity within the tumor. In cases where a stereotactic biopsy with preoperative or intraoperative imaging is planned, the areas with higher contrast enhancement are targeted to sample the highest possible grade. The accuracy of such biopsies is reported at $51 \%-83 \%$ for the highest possible grade.[22]

Surgical resection and biopsy The role of initial surgical resection versus biopsy has remained controversial in the management of patients with dLGGs without significant neurological deficits or mass effect that may demand early intervention. The infiltrative nature of dLGGs in the majority of cases and eloquent locations makes GTR challenging due to significant risk of neurological morbidity. However, biopsy, even assisted with stereotactic targeting, may not identify the highest possible grade in up to $50 \%$

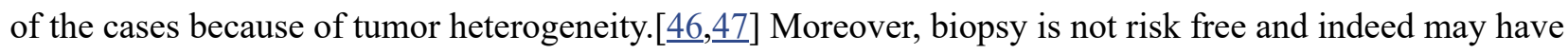
mortality/morbidity risk equating those of modern series of surgical resections[뇨] Gross or near GTR is associated with better seizure control and higher PFS and OS in addition to a lower risk of malignant transformation.[10] Chang et al. in 2008 retrospectively reviewed medical charts of 332 patients who underwent initial surgical resection of dLGGs at a single center.[13] Two hundred and sixty-nine (81.02\%) patients had seizure episodes preoperatively, of which 132 (49\%) had pharmacoresistant epilepsy. Postoperatively, $67 \%$ remained seizure free, $17 \%$ had rare seizure episodes, $8 \%$ showed improvement in the seizure activity, whereas only $9 \%$ were noted to have no improvement of the seizure episodes over a follow-up period of 1 year.[13] 
GTR was associated with better seizure control compared to subtotal resection (STR) or biopsy alone (odds ratio: $16, P=0.0064$ ). Tumor progression was identified in cases that showed seizure relapse following initial postoperative seizure control $(P=0.001)$. Sanai and Berger in 2008 reviewed the literature from 1990 to 2008 and found ten relevant studies describing surgical resection in dLGG patients. [49] The review confirmed a higher OS in patients with near-GTR. Duffau in 2008 reported a personal consecutive series of 51 patients who underwent surgical resection for insular WHO Grade II gliomas under cortico-subcortical stimulation. [무] Fifty patients underwent diagnostic studies for their presentation with seizure episodes, among which $45 \%$ of the patients revealed normal neurologic examination findings preoperatively. In the immediate postoperative period, $59 \%$ of the patients showed worsening of their condition, whereas 3-month postoperative follow-up showed $96 \%$ of the total patients returning to their baseline or an improved state of health. An $82 \%$ survival rate was reported at over a median follow-up period of 4 years.

Majchrzak et al. in 2012 prospectively analyzed the extent of resection (EOR) and assessed final outcomes in 68 patients who underwent surgical resection of hemispheric dLGGs.[51] The tumor volume and EOR were assessed by FLAIR MRI images to compare the pre- and post-operative findings, whereas dynamic susceptibility contrast perfusion MRI was used to assess rCBV.

They achieved GTR $(>95 \%)$ in $31 \%$ patients $(n=21)(85 \%-95 \%)$, STR in $19 \%(n=13)$, and partial $(<85 \%)$ resection (PTR) in $50 \%$ of the patients $(n=34)$. The EOR was statistically lower for tumors in eloquent areas or close proximity. In Cox proportional hazard analyses, the OS was predicted by age at presentation (hazard ratio [HR] 1.12, $P=0.032$ ), EOR (HR 0.96, $P=0.025)$, and $\mathrm{rCBV}(\mathrm{HR} 7.39, P=$ 0.002 ) at $5 \%$ level of significance. The estimated 5 -year OS was $100 \%$ for EOR $>80 \%$. Over a median follow-up period of 34 months, $6 \%$ mortality was observed. Similarly, the PFS was predicted by preoperative tumor volume (HR 1.01, $P=0.005$ ), postoperative tumor volume (HR 1.01, $P=0.008$ ), the EOR (HR 5.17, $P=0.001$ ), percentage of resection (HR 0.98, $P=0.004)$, and by the $\mathrm{rCBV}(\mathrm{HR} 1.70, P=$ 0.033 ) at $5 \%$ level of significance. Permanent neurologic sequelae were noticed in $6(9 \%)$ patients which showed no statistical dependence on the EOR.[51]

Jakola et al. in 2012 compared the OS in 47 patients who underwent biopsy and watchful waiting to 75 patients that underwent early surgical resection of dLGGs at two different centers with parallel cohorts. [42] The cohort undergoing biopsy and observation had a median survival of 5.9 years while median survival was not reached in the group, with the center favoring early resection. In a follow-up analysis published recently, the authors extended the follow-up to report that the OS was significantly worse in the center advocating watchful waiting with a median survival of 5.8 years compared to 14.4 years for the center that preferred early surgical resection $(P<0.01)$.[52]

A randomized controlled trial to test the efficacy of surgery for dLGG is not a practical or ethical consideration with the weight of observational studies and reviews supporting early and maximum safe resection "up front." Seizure control is greatly improved by tumor surgery,[13] and if the zone of T2FLAIR hyperintensity is eliminated on the postresection MR scan, survival is improved and malignant transformation delayed.[53] However, it must be recognized that resection cannot cure a dLGG, and minimizing operative morbidity must be the surgeon's priority. Functional regions adjacent to the tumor must be preserved by accurate identification.

This may be planned preoperatively by integrating functional MRI (FMRI) and DTI tractography information into the surgical plan. Intraoperatively, neuronavigation systems incorporating anatomical and tractographic images can guide the surgery. Adding ultrasonography and, where possible, intraoperative MRI or CT updates the preoperatively acquired images. Electrostimulation for brain mapping is the most useful method of delineating the boundaries of resection in the cortex and subjacent subcortical white matter. This requires the patient to be awake and cooperative, and this method is being used increasingly to maximize safe resections. De Witt et al. carried out a meta-analysis of 90 reports on 8091 patients.[54] 
They compared surgical resection of gliomas with and without intraoperative stimulation mapping (ISM). They demonstrated a radiologically proven GTR rate of 75\% (95\% confidence interval [CI], 66\% to $82 \%$ ) with the use of ISM compared to 58\% (95\% CI 48\%-69\%) without using ISM.[54] Of the newer technologies, only ISM has been proven to improve EOR and safety of the procedure. The rate of new neurological deficits was $3.4 \%$ compared to $8.2 \%$ with and without the use of ISM, respectively.[54]

Neuronavigation has not shown promise in increasing the EOR or increasing its safety.[55]

Adjuvant chemotherapy and radiation The more recent reported experience has supported the use of chemotherapeutics for managing dLGGs as initial therapy and as adjunct to postresection residual disease or progressive recurrent tumors. Hoang-Xuan et al. administered temozolomide (TMZ) to sixty adult patients with biopsy-proven and radiologically progressive dLGGs.[두] The tumors responded radiologically in $31 \%$ of the cases, the disease remained stable in $61 \%$ of the patients, whereas $8 \%$ of the dLGGs showed radiologic progression.

The tumors' maximum response was noticed at a median follow-up of 12 months, and chromosome $1 \mathrm{p}$ deletion was significantly associated with the tumor response $(P<0.004)$. Ricard et al. in 2007 analyzed clinical information of 107 adult patients who underwent TMZ chemotherapy for biopsy-proven dLGG with clinical or radiologic progressive disease.[57] The tumors' genetic profiles were obtained in majority of the cases for $1 \mathrm{p}, 19 \mathrm{q}$ deletion, and p53 overexpression. At a median follow-up of 2 years, $65(60.7 \%)$ patients achieved a partial or minor response on imaging, $35(32.7 \%)$ patients remained stable, and 7 (6.5\%) patients showed progression of the disease. Sixty-eight (63.5\%) patients showed clinical improvement, 34 (31.8\%) patients remained stable, whereas 5 (4.7\%) showed deteriorating clinical condition. The tumors with $1 \mathrm{p}-19 \mathrm{q}$ codeletion showed a significantly higher objective response to the therapy compared to the ones without this codeletion ( 73 vs. $50 \%, P=0.03$ ). Seizure activity in the patients was better controlled with chemotherapy even in the absence of radiological improvement.

Radiation therapy (RT) has been the adjuvant modality of convention in dLGG, but conflicting information has emerged regarding the timing and the dose. In 2005, the EORTC 22845 randomized trial reported a post-dLGG resection comparison of OS and PFS in 157 patients who underwent early RT and 157 patients in the control group who were watched radiologically without any intervention. [58] The trial showed a median PFS of 5.3 years in the early RT-group and 3.4 years in the control group (HR 0.59, $P<0.0001$ ), whereas the OS showed no significant difference for either group (HR 0.97, $P=0.872$ ). One-year followup showed better seizure control in the group undergoing early RT $(P=0.0329)$. Shaw et al. compared survival in 203 adult patients with dLGGs who underwent low- and high-dose RT.[두] One hundred and one of these patients underwent low-dose RT, while 102 underwent high-dose RT, after GTR, STRs, or biopsy. At a median follow-up period of 6.43 years, 83 (41\%) died, whereas low- or high-dose RT showed no significant difference in their impact on survival. Tumor's histologic features, size, and patients age at presentation were the significant prognostic factors recognized by multivariate analysis, and significantly higher survival was associated with younger age at presentation, in addition to tumor histology (oligodendroglioma) or oligo-rich histological components in the biopsy. Seven (5.8\%) patients showed Grade 3-5 radiation neurotoxicity. Douw et al. looked at the cognitive function over a period of 12 years in dLGG survivors who underwent RT.[60] RT had significant impact on the attention $(P=0.003)$, executive functions $(P=0.03)$, and information processing speed $(P=0.05)$ when compared to the patients who did not undergo RT.

The risk of delayed neurotoxicity following RT and the lack of survival advantage of early treatment or dose escalation have led to a tendency to withhold radiation as the first adjuvant option despite the recent advances in the planning and delivery of conformal RT. In tumors without IDH mutation or codeletion or those clinically at high risk of progression due to age, residual disease after surgery, and astrocytic histology, early RT may be beneficial, particularly those patients progressing after chemotherapy.[61] 
In 2012, the RTOG 9802 showed that administering chemotherapy to dLGG patients, in addition to RT, improved the PFS over a median follow-up of 5.9 years.[62] Adding chemotherapy to RT did not improve the OS in these patients, whereas on post hoc analysis for 2-year survivors, the combination of chemotherapy with RT showed better survival, which can be explained by delayed effectiveness. Based on the results of this trial, the current trend is to prescribe combined postoperative chemoradiation when the decision for early or late adjuvant therapy in made in a patient with dLGG.

Follow-up plan Assessment of treatment response and disease progression poses a challenge in cases of dLGGs, because of steady growth rate and limited contrast enhancement.[63] The Response Assessment in Neuro- Oncology (RANO) Working Group proposes criteria for defining the assessment of dLGG post resection and adjuvant therapies using MRI (T2/FLAIR sequence). According to RANO, the posttherapy status of the disease can be categorized in five groups; (1) Complete response, (2) partial response, (3) minor response, (4) stable disease, and (5) progression. The literature identifies no clinical trials in order to recommend an optimal follow-up imaging frequency posttreatment. The National Comprehensive Cancer Network[64] recommends imaging surveillance via MRI 3 or 6 monthly postoperative for the initial 5 years followed by yearly imaging later on. Rather than depending on the measurement tools of MRI scanners for subjective assessment of tumor dimensions, it is recommended that mean tumor diameter be calculated from semi-automated tumor contouring derived from multiple FLAIR slices to allow improved detection of subtle changes.[ㄷ]

Follow-up after surgery of dLGG should be geared to assessing the progress of any residual after sub-total resection or recurrence after GTR to preempt the progression to malignancy by adjuvant therapy and/or repeat resection.

\section{Conclusion}

The therapeutic approach to dLGG is undergoing a change from an expectant mode to a more interventional one based on the premise that the tumor will inevitably show biological progression. Recent experience suggests that malignant transformation may be delayed and survival prolonged by early surgery with maximum safe resection. For a lesion that has a proclivity for arising in eloquent regions of the brain, the safety of surgery is imperative and morbidity may be minimized by electrical mapping of the cortex and subcortical white matter in proximity in an awake patient. An alternative approach that uses newer technologies to maximize the EOR is one in which neuronavigation systems integrate FMRI to identify motor and speech cortex and fiber tracking DTI to display the eloquent cortical regions and associated white matter tracts at risk in the planning of the tumor excision. In the recent WHO classification of gliomas, genetic profile of the tumor is integrated with the histological features to arrive at a multilayered diagnosis which more accurately guides the postsurgical chemoradiation with improving patient survivals.

\section{Financial support and sponsorship}

Nil.

\section{Conflicts of interest}

There are no conflicts of interest.

\section{References}

1. Rudà R, Trevisan E, Soffietti R. Epilepsy and brain tumors. Curr Opin Oncol. 2010;22:611-20. [PubMed: 20706121]

2. Rosati A, Tomassini A, Pollo B, Ambrosi C, Schwarz A, Padovani A, et al. Epilepsy in cerebral glioma: Timing of appearance and histological correlations. J Neurooncol. 2009;93:395-400. [PubMed: 19183850] 
3. Prayson RA. Diagnostic challenges in the evaluation of chronic epilepsy-related surgical neuropathology. Am J Surg Pathol. 2010;34:e1-13. [PubMed: 20414101]

4. Klein M, Engelberts NH, van der Ploeg HM, Kasteleijn-Nolst Trenité DG, Aaronson NK, Taphoorn MJ, et al. Epilepsy in low-grade gliomas: The impact on cognitive function and quality of life. Ann Neurol. 2003;54:514-20. [PubMed: 14520665]

5. Taphoorn MJ, Klein M. Cognitive deficits in adult patients with brain tumours. Lancet Neurol. 2004;3:159-68. [PubMed: 14980531]

6. Danfors T, Ribom D, Berntsson SG, Smits A. Epileptic seizures and survival in early disease of grade 2 gliomas. Eur J Neurol. 2009;16:823-31. [PubMed: 19473357]

7. Bauman G, Lote K, Larson D, Stalpers L, Leighton C, Fisher B, et al. Pretreatment factors predict overall survival for patients with low-grade glioma: A recursive partitioning analysis. Int J Radiat Oncol Biol Phys. 1999;45:923-9. [PubMed: 10571199]

8. Bauman G, Fisher B, Watling C, Cairncross JG, Macdonald D. Adult supratentorial low-grade glioma: Long-term experience at a single institution. Int J Radiat Oncol Biol Phys. 2009;75:1401-7. [PubMed: 19395201]

9. Schiff D, Brown PD, Giannini C. Outcome in adult low-grade glioma: The impact of prognostic factors and treatment. Neurology. 2007;69:1366-73. [PubMed: 17893297]

10. Pallud J, Le Van Quyen M, Bielle F, Pellegrino C, Varlet P, Cresto N, et al. Cortical GABAergic excitation contributes to epileptic activities around human glioma. Sci Transl Med. 2014;6:244ra89. [PMCID: PMC4409113] [PubMed: 25009229]

11. Kaloshi G, Psimaras D, Mokhtari K, Dehais C, Houillier C, Marie Y, et al. Supratentorial low-grade gliomas in older patients. Neurology. 2009;73:2093-8. [PubMed: 19907009]

12. Rudà R, Bello L, Duffau H, Soffietti R. Seizures in low-grade gliomas: Natural history, pathogenesis, and outcome after treatments. Neuro Oncol. 2012;14(Suppl 4):iv55-64. [PMCID: PMC3480244] [PubMed: 23095831]

13. Chang EF, Potts MB, Keles GE, Lamborn KR, Chang SM, Barbaro NM, et al. Seizure characteristics and control following resection in 332 patients with low-grade gliomas. J Neurosurg. 2008;108:227-35. [PubMed: 18240916]

14. Hinsdale, IL: Central Brain Tumor Registry of the United States; 2010. Central Brain Tumor Registry of the United States. Central Brain Tumor Registry of the United States Statistical Report: Primary Brain and Central Nervous System Tumors Diagnosed in the United States in 2004-2006.

15. Yeh SA, Lee TC, Chen HJ, Lui CC, Sun LM, Wang CJ, et al. Treatment outcomes and prognostic factors of patients with supratentorial low-grade oligodendroglioma. Int J Radiat Oncol Biol Phys. 2002;54:1405-9. [PubMed: 12459363]

16. Wessels PH, Weber WE, Raven G, Ramaekers FC, Hopman AH, Twijnstra A, et al. Supratentorial grade II astrocytoma: Biological features and clinical course. Lancet Neurol. 2003;2:395-403. [PubMed: 12849117]

17. Lebrun C, Fontaine D, Ramaioli A, Vandenbos F, Chanalet S, Lonjon M, et al. Long-term outcome of oligodendrogliomas. Neurology. 2004;62:1783-7. [PubMed: 15159478]

18. Recht LD, Lew R, Smith TW. Suspected low-grade glioma: Is deferring treatment safe? Ann Neurol. 1992;31:431-6. [PubMed: 1586143] 
19. Potts MB, Smith JS, Molinaro AM, Berger MS. Natural history and surgical management of incidentally discovered low-grade gliomas. J Neurosurg. 2012;116:365-72. [PubMed: 21999317]

20. Guillevin R, Herpe G, Verdier M, Guillevin C. Low-grade gliomas: The challenges of imaging. Diagn Interv Imaging. 2014;95:957-63. [PubMed: 25195186]

21. Fouke SJ, Benzinger T, Gibson D, Ryken TC, Kalkanis SN, Olson JJ, et al. The role of imaging in the management of adults with diffuse low grade glioma: A systematic review and evidence-based clinical practice guideline. J Neurooncol. 2015;125:457-79. [PubMed: 26530262]

22. Pouratian N, Asthagiri A, Jagannathan J, Shaffrey ME, Schiff D. Surgery insight: The role of surgery in the management of low-grade gliomas. Nat Clin Pract Neurol. 2007;3:628-39. [PubMed: 17982433]

23. Pirzkall A, Nelson SJ, McKnight TR, Takahashi MM, Li X, Graves EE, et al. Metabolic imaging of low-grade gliomas with three-dimensional magnetic resonance spectroscopy. Int J Radiat Oncol Biol Phys. 2002;53:1254-64. [PubMed: 12128127]

24. Gaudino S, Russo R, Verdolotti T, Caulo M, Colosimo C. Advanced MR imaging in hemispheric lowgrade gliomas before surgery; the indications and limits in the pediatric age. Childs Nerv Syst. 2016;32:1813-22. [PubMed: 27659824]

25. Alam MS, Sajjad Z, Hafeez S, Akhter W. Magnetic resonance spectroscopy in focal brain lesions. J Pak Med Assoc. 2011;61:540-3. [PubMed: 22204206]

26. Bisdas S, Ritz R, Bender B, Braun C, Pfannenberg C, Reimold M, et al. Metabolic mapping of gliomas using hybrid MR-PET imaging: Feasibility of the method and spatial distribution of metabolic changes. Invest Radiol. 2013;48:295-301. [PubMed: 23296081]

27. Zhang L, Min Z, Tang M, Chen S, Lei X, Zhang X, et al. The utility of diffusion MRI with quantitative ADC measurements for differentiating high-grade from low-grade cerebral gliomas: Evidence from a meta-analysis. J Neurol Sci. 2017;373:9-15. [PubMed: 28131237]

28. Dimou S, Battisti RA, Hermens DF, Lagopoulos J. A systematic review of functional magnetic resonance imaging and diffusion tensor imaging modalities used in presurgical planning of brain tumour resection. Neurosurg Rev. 2013;36:205-14. [PubMed: 23187966]

29. Server A, Graff BA, Josefsen R, Orheim TE, Schellhorn T, Nordhøy W, et al. Analysis of diffusion tensor imaging metrics for gliomas grading at 3 T. Eur J Radiol. 2014;83:e156-65. [PubMed: 24457139]

30. Ma L, Song ZJ. Differentiation between low-grade and high-grade glioma using combined diffusion tensor imaging metrics. Clin Neurol Neurosurg. 2013;115:2489-95. [PubMed: 24183513]

31. Usinskiene J, Ulyte A, Bjørnerud A, Venius J, Katsaros VK, Rynkeviciene R, et al. Optimal differentiation of high- and low-grade glioma and metastasis: A meta-analysis of perfusion, diffusion, and spectroscopy metrics. Neuroradiology. 2016;58:339-50. [PubMed: 26767528]

32. Mandonnet E, Delattre JY, Tanguy ML, Swanson KR, Carpentier AF, Duffau H, et al. Continuous growth of mean tumor diameter in a subset of grade II gliomas. Ann Neurol. 2003;53:524-8. [PubMed: 12666121]

33. Pallud J, Blonski M, Mandonnet E, Audureau E, Fontaine D, Sanai N, et al. Velocity of tumor spontaneous expansion predicts long-term outcomes for diffuse low-grade gliomas. Neuro Oncol. 2013;15:595-606. [PMCID: PMC3635513] [PubMed: 23393207]

34. Pignatti F, van den Bent M, Curran D, Debruyne C, Sylvester R, Therasse P, et al. Prognostic factors for survival in adult patients with cerebral low-grade glioma. J Clin Oncol. 2002;20:2076-84. [PubMed: 11956268] 
35. Yan H, Parsons DW, Jin G, McLendon R, Rasheed BA, Yuan W, et al. IDH1 and IDH2 mutations in gliomas. N Engl J Med. 2009;360:765-73. [PMCID: PMC2820383] [PubMed: 19228619]

36. Houillier C, Wang X, Kaloshi G, Mokhtari K, Guillevin R, Laffaire J, et al. IDH1 or IDH2 mutations predict longer survival and response to temozolomide in low-grade gliomas. Neurology. 2010;75:1560-6. [PubMed: 20975057]

37. Ricard D, Idbaih A, Ducray F, Lahutte M, Hoang-Xuan K, Delattre JY, et al. Primary brain tumours in adults. Lancet. 2012;379:1984-96. [PubMed: 22510398]

38. Wiestler B, Capper D, Holland-Letz T, Korshunov A, von Deimling A, Pfister SM, et al. ATRX loss refines the classification of anaplastic gliomas and identifies a subgroup of IDH mutant astrocytic tumors with better prognosis. Acta Neuropathol. 2013;126:443-51. [PubMed: 23904111]

39. Labussière M, Boisselier B, Mokhtari K, Di Stefano AL, Rahimian A, Rossetto M, et al. Combined analysis of TERT, EGFR, and IDH status defines distinct prognostic glioblastoma classes. Neurology. 2014;83:1200-6. [PubMed: 25150284]

40. Brat DJ, Verhaak RG, Aldape KD, Yung WK, Salama SR, et al. Cancer Genome Atlas Research Network. Comprehensive, integrative genomic analysis of diffuse lower-grade gliomas. N Engl J Med. 2015;372:2481-98. [PMCID: PMC4530011] [PubMed: 26061751]

41. Scott JN, Brasher PM, Sevick RJ, Rewcastle NB, Forsyth PA. How often are nonenhancing supratentorial gliomas malignant? A population study. Neurology. 2002;59:947-9. [PubMed: 12297589]

42. Jakola AS, Myrmel KS, Kloster R, Torp SH, Lindal S, Unsgård G, et al. Comparison of a strategy favoring early surgical resection vs. a strategy favoring watchful waiting in low-grade gliomas. JAMA. 2012;308:1881-8. [PubMed: 23099483]

43. Duffau H. Lessons from brain mapping in surgery for low-grade glioma: Insights into associations between tumour and brain plasticity. Lancet Neurol. 2005;4:476-86. [PubMed: 16033690]

44. Lote K, Stenwig AE, Skullerud K, Hirschberg H. Prevalence and prognostic significance of epilepsy in patients with gliomas. Eur J Cancer. 1998;34:98-102. [PubMed: 9624245]

45. Revesz T, Scaravilli F, Coutinho L, Cockburn H, Sacares P, Thomas DG, et al. Reliability of histological diagnosis including grading in gliomas biopsied by image-guided stereotactic technique. Brain. 1993;116(Pt 4):781-93. [PubMed: 8353708]

46. Jackson RJ, Fuller GN, Abi-Said D, Lang FF, Gokaslan ZL, Shi WM, et al. Limitations of stereotactic biopsy in the initial management of gliomas. Neuro Oncol. 2001;3:193-200. [PMCID: PMC1920616] [PubMed: 11465400]

47. Muragaki Y, Chernov M, Maruyama T, Ochiai T, Taira T, Kubo O, et al. Low-grade glioma on stereotactic biopsy: How often is the diagnosis accurate? Minim Invasive Neurosurg. 2008;51:275-9. [PubMed: 18855292]

48. Capelle L, Fontaine D, Mandonnet E, Taillandier L, Golmard JL, Bauchet L, et al. Spontaneous and therapeutic prognostic factors in adult hemispheric world health organization grade II gliomas: A series of 1097 cases: Clinical article. J Neurosurg. 2013;118:1157-68. [PubMed: 23495881]

49. Sanai N, Berger MS. Glioma extent of resection and its impact on patient outcome. Neurosurgery. 2008;62:753-64. [PubMed: 18496181]

50. Duffau H. A personal consecutive series of surgically treated 51 cases of insular WHO grade II glioma: Advances and limitations. J Neurosurg. 2009;110:696-708. [PubMed: 19133753] 
51. Majchrzak K, Kaspera W, Bobek-Billewicz B, Hebda A, Stasik-Pres G, Majchrzak H, et al. The assessment of prognostic factors in surgical treatment of low-grade gliomas: A prospective study. Clin Neurol Neurosurg. 2012;114:1135-44. [PubMed: 22425370]

52. Jakola AS, Skjulsvik AJ, Myrmel KS, Sjåvik K, Unsgård G, Torp SH, et al. Surgical resection versus watchful waiting in low-grade gliomas. Ann Oncol. 2017;28:1942-8. [PMCID: PMC5834105] [PubMed: 28475680]

53. McGirt MJ, Chaichana KL, Attenello FJ, Weingart JD, Than K, Burger PC, et al. Extent of surgical resection is independently associated with survival in patients with hemispheric infiltrating low-grade gliomas. Neurosurgery. 2008;63:700-7. [PubMed: 18981880]

54. De Witt Hamer PC, Robles SG, Zwinderman AH, Duffau H, Berger MS. Impact of intraoperative stimulation brain mapping on glioma surgery outcome: A meta-analysis. J Clin Oncol. 2012;30:2559-65. [PubMed: 22529254]

55. Willems PW, Taphoorn MJ, Burger H, Berkelbach van der Sprenkel JW, Tulleken CA. Effectiveness of neuronavigation in resecting solitary intracerebral contrast-enhancing tumors: A randomized controlled trial. J Neurosurg. 2006;104:360-8. [PubMed: 16572647]

56. Hoang-Xuan K, Capelle L, Kujas M, Taillibert S, Duffau H, Lejeune J, et al. Temozolomide as initial treatment for adults with low-grade oligodendrogliomas or oligoastrocytomas and correlation with chromosome 1p deletions. J Clin Oncol. 2004;22:3133-8. [PubMed: 15284265]

57. Ricard D, Kaloshi G, Amiel-Benouaich A, Lejeune J, Marie Y, Mandonnet E, et al. Dynamic history of low-grade gliomas before and after temozolomide treatment. Ann Neurol. 2007;61:484-90. [PubMed: 17469128]

58. van den Bent MJ, Afra D, de Witte O, Ben Hassel M, Schraub S, Hoang-Xuan K, et al. Long-term efficacy of early versus delayed radiotherapy for low-grade astrocytoma and oligodendroglioma in adults: The EORTC 22845 randomised trial. Lancet. 2005;366:985-90. [PubMed: 16168780]

59. Shaw E, Arusell R, Scheithauer B, O’Fallon J, O’Neill B, Dinapoli R, et al. Prospective randomized trial of low- versus high-dose radiation therapy in adults with supratentorial low-grade glioma: Initial report of a North Central Cancer Treatment Group/Radiation Therapy Oncology Group/Eastern Cooperative Oncology Group study. J Clin Oncol. 2002;20:2267-76. [PubMed: 11980997]

60. Douw L, Klein M, Fagel SS, van den Heuvel J, Taphoorn MJ, Aaronson NK, et al. Cognitive and radiological effects of radiotherapy in patients with low-grade glioma: Long-term follow-up. Lancet Neurol. 2009;8:810-8. [PubMed: 19665931]

61. Duffau H, Taillandier L. New concepts in the management of diffuse low-grade glioma: Proposal of a multistage and individualized therapeutic approach. Neuro Oncol. 2015;17:332-42.

[PMCID: PMC4483091] [PubMed: 25087230]

62. Shaw EG, Wang M, Coons SW, Brachman DG, Buckner JC, Stelzer KJ, et al. Randomized trial of radiation therapy plus procarbazine, lomustine, and vincristine chemotherapy for supratentorial adult lowgrade glioma: Initial results of RTOG 9802. J Clin Oncol. 2012;30:3065-70. [PMCID: PMC3732006] [PubMed: 22851558]

63. van den Bent MJ, Wefel JS, Schiff D, Taphoorn MJ, Jaeckle K, Junck L, et al. Response assessment in neuro-oncology (a report of the RANO group): Assessment of outcome in trials of diffuse low-grade gliomas. Lancet Oncol. 2011;12:583-93. [PubMed: 21474379]

64. Forst DA, Nahed BV, Loeffler JS, Batchelor TT. Low-grade gliomas. Oncologist. 2014;19:403-13. [PMCID: PMC3983820] [PubMed: 24664484] 
65. Rees J, Watt H, Jäger HR, Benton C, Tozer D, Tofts $P$, et al. Volumes and growth rates of untreated adult low-grade gliomas indicate risk of early malignant transformation. Eur J Radiol. 2009;72:54-64. [PubMed: 18632238]

Figures and Tables 


\section{Figure 1}

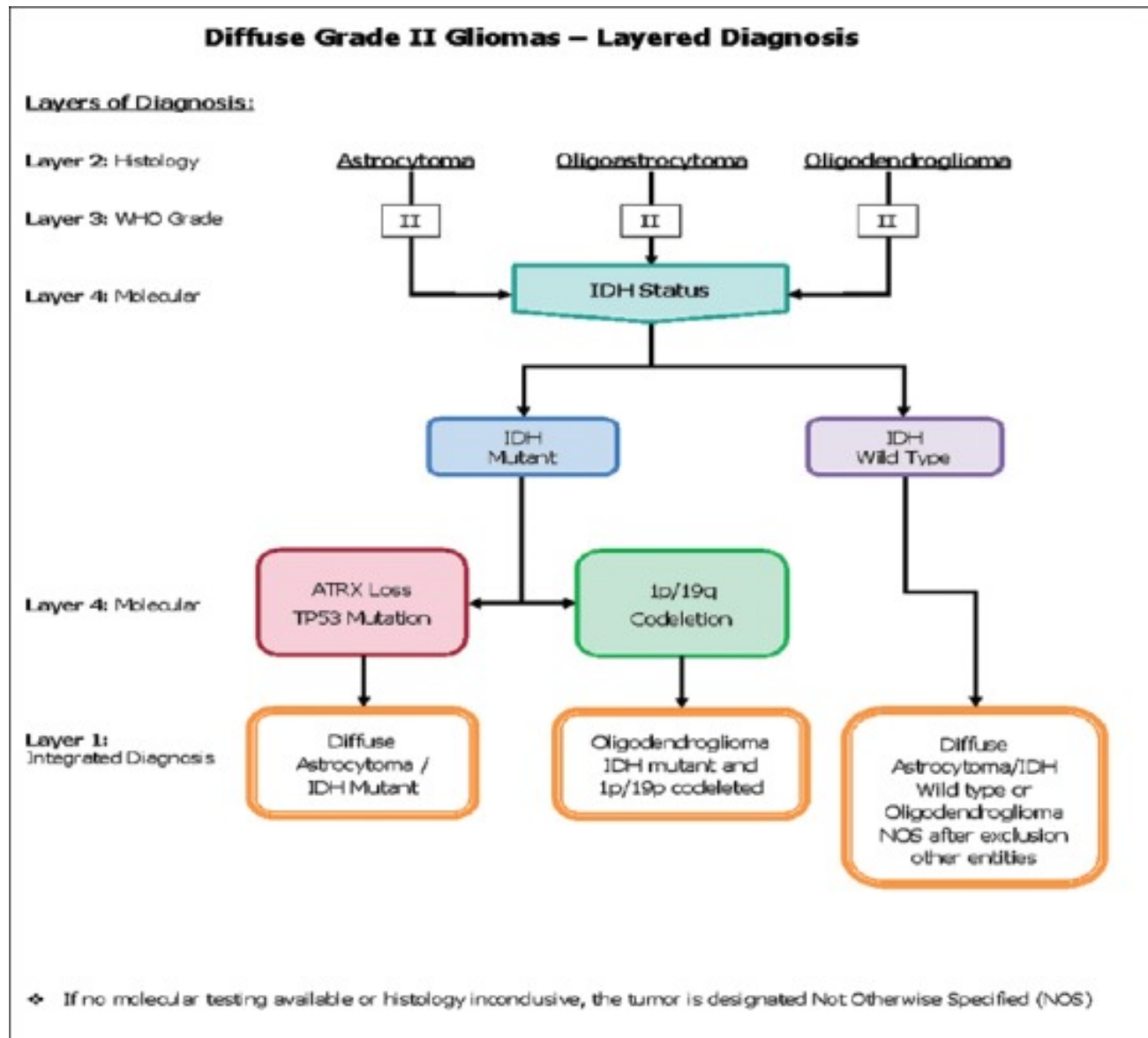

Open in a separate window

Layered diagnosis of low-grade glioma according to the WHO classification

Articles from Asian Journal of Neurosurgery are provided here courtesy of Wolters Kluwer -- Medknow Publications 